\title{
Application of Remote Follow-Up Via the WeChat Platform for Patients who Underwent Congenital Cardiac Surgery During the COVID-19 Epidemic
}

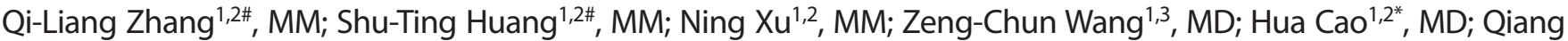 \\ Chen ${ }^{1,2,3^{*}}, \mathrm{MD}$
}

DOI: 10.21470/1678-9741-2020-0256

\begin{abstract}
Objective: To investigate the effect of WeChat-based telehealth services on the postoperative follow-up of children who underwent congenital heart surgery during the COVID-19 epidemic.

Methods: This study retrospectively analyzed the clinical and family data of 108 children who underwent congenital heart surgery and underwent remote follow-up via the WeChat platform from December 2019 to March 2020 in our hospital.

Results: During the follow-up period, the WeChat platform was used to refer 8 children with respiratory infection symptoms to local hospitals for treatment. Two children with poor incision healing were healed after we used the WeChat platform to guide the parents in dressing the wounds on a regular basis at home. Nutritional guidance was given via the WeChat platform to 13
\end{abstract}

patients with poor growth and development. The psychological evaluation results of the parents showed that the median (range) SDS score was 43 (34-59), and 7 parents $(6.5 \%)$ were classified as depressed; the median (range) SAS score was 41 (32-58), and 12 parents $(11.1 \%)$ were classified as having mild anxiety.

Conclusion: The use of WeChat-based telehealth services was effective for the remote postoperative follow-up of children who underwent congenital cardiac surgery during the COVID-19 epidemic. Providing WeChat-based telehealth services can reduce the amount of travel required for these children and their families, which is helpful for controlling and preventing the spread of COVID-19.

Keywords: COVID-19. Social Media. Attitude to Health. Cardiovascular System. China, Severe Acute Respiratory coronavirus 2.

\begin{tabular}{ll}
\hline \multicolumn{2}{l}{ Abbreviations, acronyms \& symbols } \\
\hline CHD & $=$ Congenital heart disease \\
COVID-19 & $=$ Coronavirus disease 2019 \\
SARS & $=$ Severe acute respiratory syndrome \\
SAS & $=$ Self-Rating Anxiety Scale \\
SDS & $=$ Self-Rating Depression Scale \\
\hline
\end{tabular}

\section{INTRODUCTION}

Recently, a new coronavirus disease (COVID-19) emerged and spread quickly in China and around the world ${ }^{[1]}$. Although strong measures have been taken to control the virus, there were still a large number of people infected, and some of them died because of ineffective treatment ${ }^{[2-6]}$. During this particular

'Department of Cardiac Surgery, Fujian Maternity and Child Health Hospital, Affiliated Hospital of Fujian Medical University, Fuzhou, China.

${ }^{2}$ Fujian Key Laboratory of Women and Children's Critical Diseases Research, Fujian Maternity and Child Health Hospital, Fuzhou, China.

${ }^{3}$ Department of Cardiovascular Surgery, Union Hospital, Fujian Medical University, Fuzhou, China.

\#These authors contributed equally to this study and shared the first authorship. *These authors shared the corresponding authorship.

This study was carried out at the Department of Cardiac Surgery, Fujian Maternity and Child Health Hospital, Affiliated Hospital of Fujian Medical University, Fuzhou, China. period, people with congenital heart disease (CHD) still had to undergo surgical correction and needed close medical monitoring. The poor physical quality and immune functions of children who underwent congenital cardiac surgery made them more susceptible to respiratory infection than normal people. However, regular monitoring of these patients was required to assess their postoperative recovery. Due to the uneven distribution of medical resources, many patients living in remote areas have to travel to hospitals in central cities in China to undergo cardiac surgery. There was a high risk of respiratory infection in the hospital and while traveling to the hospital, as this travel might increase population mobility and the spread of the disease. For patients, if they came to the hospital for a follow-up visit as scheduled, they would face the risk of being infected by the virus; if they did not come to the hospital, they

Correspondence Address:

Qiang Chen

iD.ps://orcid.org/0000-0003-3768-9000

Department of Cardiac Surgery, Fujian Maternity and Child Health Hospital,

Affiliated Hospital of Fujian Medical University, Daoshan Road 18\#, Fuzhou, Fujian,

China - Zip code: 350004

E-mail: chenqiang2228@163.com 
would worry that any subsequent heart problems might not be addressed in time. These options led to a heavy mental burden for the parents. Therefore, providing effective telehealth services for the postoperative follow-up of children who underwent congenital cardiac surgery has become extremely important during the COVID-19 epidemic. The purpose of this study was to explore the effects of using the WeChat platform on the remote postoperative follow-up of these patients during the epidemic.

\section{METHODS}

The present study was approved by the ethics committee of Fujian Medical University, China, and adhered to the tenets of the Declaration of Helsinki. Additionally, all parents signed the consent form before participating in the study.

\section{Patients}

This study retrospectively analyzed the clinical and family data of 108 children who underwent congenital cardiac surgery and remote follow-up via the WeChat platform from December 2019 to March 2020 at our hospital. All patients' underlying diseases were assessed: 32 cases of atrial septal defect, 46 cases of ventricular septal defect, 13 cases of patent ductus arteriosus, 12 cases of pulmonary stenosis, and 5 cases of tetralogy of Fallot. Other relevant clinical data are shown in Table 1.

\section{WeChat-Based Telehealth Services}

The content of the telehealth service provided via the WeChat platform mainly included two parts: the education module and the answering and solving questions module. The education module included relevant knowledge about $\mathrm{CHD}$, postoperative care and nutrition, and the management of related postoperative complications. Parents could review and learn these materials at their convenience. Questionnaires were distributed through the WeChat platform to assess the psychological status of the parents of children who underwent congenital cardiac surgery during the COVID-19 epidemic. This questionnaire mainly included the Self-Rating Depression Scale (SDS) and the Self-Rating Anxiety Scale (SAS). In addition to the structured questionnaire, parents were allowed to express any other concerns. In the answering and solving questions module, one medical staff member was available from 8 am to $21 \mathrm{pm}$ every day to answer parents' questions, send reminders and supervise regular outpatient reviews, and remind parents about the possibility of postoperative complications due to delayed treatment. The medical staff also encouraged the family members to actively discuss the care experience with each other in the WeChat group.

\section{Research tools}

1.SDS was applied in this study ${ }^{[7]}$. This scale consists of 20 items, including 10 negative symptoms and 10 positive symptoms. The items assess the respondent's mood, body discomfort symptoms, spiritual movement, behavior, and psychological symptoms. The response options for each item range from 1 to 4 , with higher scores indicating a higher frequency of symptoms (the items were reverse scored to assess the frequency of negative symptoms). The standardized score was obtained by multiplying the scores by 1.25 and rounding the result. Normally, the maximum score was 41 and the maximum standardized total score was 53 . Higher scores indicate a trend towards more significant depression. Grade description: $<50$ indicates normal, 50 to 59 indicates mild depression, 60 to 69 indicates moderate depression, and $\geq 70$ indicates severe depression.

2. SAS was applied in this study ${ }^{[8]}$. This scale mainly evaluated the subjective patients'feeling of anxiety, being a selfreported tool. SAS has been extensively applied in clinics and is characterized by high reliability and validity. The scale included 15 items with negative words and five items with positive words. The response options for each item range from 1 to 4 , with higher scores indicating a higher frequency of symptoms (the items were reverse scored to assess the frequency of the positive symptoms). The total score was obtained by summing the scores of all items. The standardized score was obtained by multiplying the total score by 1.25 and rounding the result. The mean value of the standardized score is 50 . Grade description: $<50$ indicates normal, 50 to 59 indicates mild anxiety, 60 to 69 indicates moderate anxiety, and $\geq 70$ indicates severe anxiety.

\section{RESULTS}

A total of 108 children who underwent congenital cardiac surgery participated in this study. The median age of the children was 2.8 years, and ages ranged from 1 month to 9 years. The median weight was $12.4 \mathrm{~kg}$, and the weights ranged from 3.2 $\mathrm{kg}$ to $37.6 \mathrm{~kg}$. A total of $70.4 \%(76 / 108)$ of the children lived in rural areas, and $57.4 \%(62 / 108)$ of the parents had high school education or lower education level (Table 1).

Eight children with respiratory infection symptoms were referred to local hospitals via the WeChat platform. These patients were diagnosed with community-acquired pneumonia at the local hospital through symptoms, signs and tests and were also excluded from specific bacterial or viral infections. They were easily cured with conventional drugs. Two children with poor incision healing were healed after we used WeChat to guide the parents in dressing the wounds on a regular basis at home. Nutritional guidance was given via the WeChat platform to 13 patients with poor growth and development. No child suffered from arrhythmia or heart failure (Table 2). The parents' psychological evaluations showed that the median (range) SDS score was 43 (34-59), and 7 parents (6.5\%) were classified as depressed; the median (range) SAS score was 41 (32-58), and 12 parents (11.1\%) were classified as having mild anxiety (Table 3).

\section{DISCUSSION}

CHD is the most common congenital structural deformity; $2-3 \%$ of newborns suffer from CHD every year, and this deformity may be life-threatening if not treated ${ }^{[9-11]}$. Surgical correction is the main method to treat CHD. Children with congenital cardiac surgery are usually affected by cardiac malformations, and their physical and immune functions are often worse than those of normal people. Therefore, children who undergo congenital cardiac surgery often need close follow-up and regular 
Table 1. Patient demographics or patient characteristics.

\begin{tabular}{l|c}
\hline \multicolumn{1}{c|}{ Item } & \\
\hline Number & 108 \\
\hline Age, median (range) & 2.8 years (1 month-9 years) \\
\hline Weight, median (range) & $12.4(3.2-37.6) \mathrm{kg}$ \\
\hline Disease & \\
\hline Atrial septal defect & 32 \\
\hline Ventricular septal defect & 46 \\
\hline Patent ductus arteriosus & 13 \\
\hline Pulmonary stenosis & 12 \\
\hline Tetralogy of Fallot & 5 \\
\hline Parents' age, median (range) & 29 years (22-43) years \\
\hline Parents' education level & 20 \\
\hline Under high school & 42 \\
\hline High school & 31 \\
\hline Junior college & 15 \\
\hline Bachelor degree or higher & 76 \\
\hline Living condition & \\
\hline Rural area & \\
\hline City & \\
\hline
\end{tabular}

Table 2. Postoperative complications of children.

\begin{tabular}{l|c}
\hline \multicolumn{1}{c|}{ Item } & \\
\hline Respiratory infections & 8 \\
\hline Poor growth and development & 13 \\
\hline Poor incision healing & 2 \\
\hline Arrhythmia & 0 \\
\hline Heart failure & 0 \\
\hline
\end{tabular}

monitoring to assess their physical condition and postoperative cardiac recovery after discharge.

The COVID-19 epidemic broke out in China and spread rapidly across the country and the world in December 2019 [12]. To effectively prevent and control the epidemic, the Chinese government has taken strict measures such as blocking cities and roads to limit the flow of people, encouraging all residents to stay at home as much as possible and restricting social contact to cut transmission routes and reduce the spread of the virus ${ }^{[13]}$. During this epidemic period, only a limited portion of the urban public transport system was working, and all interprovincial public transport routes were discontinued. However, advanced medical care is mainly concentrated in some large cities in China, and the primary health facilities are relatively scarce; thus, the number of
Table 3. Parents' psychological status.

\begin{tabular}{c|c}
\hline \multicolumn{1}{|c}{ Item } & \\
\hline SDS score, median (range) & $43(34-59)$ \\
\hline Normal (score: $<50)$ & 101 \\
\hline Mild depression (score: $50-59)$ & 7 \\
\hline Moderate depression (score: $60-69)$ & 0 \\
\hline Severe depression (score: $>70)$ & 0 \\
\hline SAS score, median (range) & $41(32-58)$ \\
\hline Normal (score: $<50)$ & 96 \\
\hline Mild anxiety (score: $50-59)$ & 12 \\
\hline Moderate anxiety (score: $60-69)$ & 0 \\
\hline Severe anxiety (score: $>70)$ & 0 \\
\hline
\end{tabular}

doctors able to perform cardiac surgery is low. However, most patients with CHD live in rural areas, and the patients'parents mostly have secondary education or lower education level, so they have little knowledge about CHD and are unable to fully understand the information about the disease during hospitalization. In this study, $70.4 \%$ of the children lived in rural areas far from the city, and $57.4 \%$ of the patients' parents had secondary education or lower education level. Many parents might feel pressure and anxiety about postoperative family care and find it difficult to deal with unexpected events. During this epidemic, it was very inconvenient for people to travel for medical treatment, especially people living in rural areas, and this travelling might increase the risk of virus infection. A lack of medical knowledge and difficulty in seeking medical support would greatly increase the pressure and anxiety among the parents of children who underwent congenital cardiac surgery. Therefore, it was particularly important to implement a new method to remote follow-up with these patients and to improve telehealth services, which could provide patients with medical service support and facilitate the prevention and control of COVID-19.

Smartphones have been widely used in recent years, especially in China ${ }^{[14]}$. Many different social media platforms have been widely used in health management and in diseaserelated education ${ }^{[15,16]}$. Unlike other social media platforms, the WeChat platform can be used for graphs, text, audio, video and so on. It is a popular, convenient, interactive and intuitive way of exchanging information, and thus, it is an important platform for maximizing information coverage and effectiveness ${ }^{[17]}$. In addition, health education via the WeChat platform has been found to be very effective in saving time, reducing economic costs, improving treatment adherence, reducing complications, increasing follow-up rates and improving patients' conditions $^{[18-20]}$. During the COVID-19 epidemic, we provided remote follow-up and telehealth services through the WeChat platform for children who underwent congenital cardiac surgery. The parents of these children could acquire knowledge from the WeChat education module anytime and anywhere according to 
their needs. When there was a problem, they could consult the medical staff through WeChat so that they could obtain timely and effective answers from professionals. In this study, 8 children with respiratory infections were referred to local hospitals after receiving timely advice via the WeChat platform. Two children with poor incision healing were cured after we used WeChat video to guide the parents in dressing the wounds at home. We provided nutritional guidance through the WeChat platform to 13 patients with poor growth and development. In this way, the parents could obtain medical support without leaving home, which reduced the need to travel for medical care during the COVID-19 epidemic.

Infectious diseases not only affect the physical health of patients but also affect the mental health and well-being of uninfected people ${ }^{[21]}$. Previous studies have shown that an increase in the prevalence of infectious diseases, such as severe acute respiratory syndrome (SARS), leads to increases in the levels of anxiety, depression and stress in the population ${ }^{[22-23]}$. Studies have also shown that people with health problems and their families who needed to go to the clinic were vulnerable to anxiety and depression, and the panic and anxiety caused by the pandemic of COVID-19 would further exaggerate these conditions ${ }^{[24]}$. During this epidemic, due to the inconvenience of traveling for hospital visits, patients could not return to the hospital for timely follow-up visits. The parents were worried about the cardiac recovery of the child, especially when the child had symptoms such as respiratory tract infection, poor growth and development, and poor wound healing. Through the WeChat platform, parents could directly contact the medical staff and easily obtain support from doctors, thus alleviating their concerns in a timely manner and reducing their anxiety and depression. Additionally, this method allowed us to provide psychological counseling and support to parents with high levels of stress or anxiety. In this study, most parents did not experience anxiety and depression, and those who experienced anxiety and depression showed only mild symptoms.

This article has some limitations. First, this study was a singlecenter retrospective study with a small number of cases. Second, we did not have a control group, so this was a descriptive study. Third, this was a study performed during an epidemic, and the results might not apply to other periods or might require further elaboration. Fourth, we did not discuss the relationship between parents' psychological status and the postoperative outcomes, patient's age, or patient's injuries in this study.

\section{CONCLUSION}

During the COVID-19 epidemic, remote follow-up and telehealth services through the WeChat platform for children who underwent congenital cardiac surgery can provide a timely assessment of the physical status of children, and this platform allows practitioners respond to problems in the process of family care and provide guidance for home care, which relieves stress, anxiety and depression of the parents of these patients. This method can also reduce the need for travel for these children and their families, which is helpful for controlling and preventing the spread of COVID-19.
Financial support: This study was supported by the National Key Research and Development Program of China (grant no. 2016YFC1301900).

\section{No conflict of interest.}

Authors' roles \& responsibilities

Q-LZ Substantial contributions to the conception or design of the work; or the acquisition, analysis or interpretation of data for the work; drafting the work or revising it critically for important intellectual content; final approval of the version to be published

S-TH Substantial contributions to the conception or design of the work; or the acquisition, analysis or interpretation of data for the work; final approval of the version to be published

NX Substantial contributions to the conception or design of the work; or the acquisition, analysis or interpretation of data for the work; final approval of the version to be published

Z-CW Substantial contributions to the conception or design of the work; or the acquisition, analysis or interpretation of data for the work; final approval of the version to be published

$\mathrm{HC}$ Substantial contributions to the conception or design of the work; or the acquisition, analysis or interpretation of data for the work; drafting the work or revising it critically for important intellectual content; final approval of the version to be published

QC Substantial contributions to the conception or design of the work; or the acquisition, analysis or interpretation of data for the work; final approval of the version to be published

\section{REFERENCES}

1. Zhu N, Zhang D, Wang W, Li X, Yang B, Song J, et al. A novel Coronavirus from patients with pneumonia in China, 2019. N Engl J Med. 2020;382(8):727-33. doi:10.1056/NEJMoa2001017.

2. Xiao H, Zhang Y, Kong D, Li S, Yang N. The effects of social support on sleep quality of medical staff treating patients with Coronavirus disease 2019 (COVID-19) in January and February 2020 in China. Med Sci Monit. 2020;26:e923549. doi:10.12659/MSM.923549.

3. Chen Y, Li Z, Zhang YY, Zhao WH, Yu ZY. Maternal health care management during the outbreak of coronavirus disease 2019. J Med Virol. 2020;92(7):731-9. doi:10.1002/jmv.25787.

4. Hui DS, I Azhar E, Madani TA, Ntoumi F, Kock R, Dar O, et al. The continuing 2019-nCoV epidemic threat of novel coronaviruses to global health - The latest 2019 novel coronavirus outbreak in Wuhan, China. Int J Infect Dis. 2020;91:264-6. doi:10.1016/j.ijid.2020.01.009.

5. Huang C, Wang Y, Li X, Ren L, Zhao J, Hu Y, et al. Clinical features of patients infected with 2019 novel coronavirus in Wuhan, China. Lancet. 2020;395(10223):497-506. Erratum in: Lancet. 2020;: doi:10.1016/S01406736(20)30183-5.

6. Mahase E. China coronavirus: what do we know so far? BMJ. 2020;368:m308. doi:10.1136/bmj.m308.

7. Zung WW. A self-rating depression scale. Arch Gen Psychiatry. 1965;12:63-70. doi:10.1001/archpsyc.1965.01720310065008.

8. Zung WW. A rating instrument for anxieties orders. Psychosomatics. 1971;12(6): 371-9. doi:10.1016/s0033-3182(71)71479-0.

9. Hoffman JI, Kaplan S. The incidence of congenital heart disease. J Am 
Coll Cardiol. 2002;39(12):1890-900. doi:10.1016/s0735-1097(02)01886-7. 10. Mazer P, Gischler SJ, Koot HM, Tibboel D, van Dijk M, Duivenvoorden HJ. Impact of a child with congenital anomalies on parents (ICCAP) questionnaire; a psychometric analysis. Health Qual Life Outcomes. 2008;6:102. doi:10.1186/1477-7525-6-102.

11. Eskedal L, Hagemo PS, Eskild A, Aamodt G, Seiler KS, Thaulow E. Survival after surgery for congenital heart defects: does reduced early mortality predict improved long-term survival? Acta Paediatr. 2005;94(4):438-43. doi:10.1111/j.1651-2227.2005.tb01915.x.

12. Chen S, Yang J, Yang W, Wang C, Bärnighausen T. COVID-19 control in China during mass population movements at New Year. Lancet. 2020;395(10226):764-6. doi:10.1016/S0140-6736(20)30421-9.

13. Liu J, Zheng X, Chai S, Lei M, Feng Z, Zhang X, Lopez V. Effects of using WeChat-assisted perioperative care instructions for parents of pediatric patients undergoing day surgery for herniorrhaphy. Patient Educ Couns. 2018;101(8):1433-8. doi:10.1016/j.pec.2018.02.010.

14. Mihalko MC. We need to talk: the impact on care when electronic communication replaces verbal communication between clinicians. J Pediatr Nurs. 2015;30(4):626-7. doi:10.1016/j.pedn.2015.04.002.

15. Yang $P$, Lo W, He ZL, Xiao XM. Medical nutrition treatment of women with gestational diabetes mellitus by a telemedicine system based on smartphones. J Obstet Gynaecol Res. 2018;44(7):1228-34. doi:10.1111/ jog.13669.

16. Feng S, Liang Z, Zhang R, Liao W, Chen Y, Fan Y, et al. Effects of mobile phone WeChat services improve adherence to corticosteroid nasal spray treatment for chronic rhinosinusitis after functional endoscopic sinus surgery: a 3-month follow-up study. Eur Arch Otorhinolaryngol. 2017;274(3):1477-85. doi:10.1007/s00405-016-4371-0.
17. Lyu KX, Zhao J, Wang B, Xiong GX, Yang WQ, Liu QH, et al. Smartphone application WeChat for clinical follow-up of discharged patients with head and neck tumors: a randomized controlled trial. Chin Med J (Engl). 2016;129(23):2816-23. doi:10.4103/0366-6999.194635.

18. Ma ZH, Xie H. Discussion on the development of medical service model based on WeChat/micro-blog. Chin Health Manag. 2014;2(3):238-9.

19. Li XH, Zhu QL. Application of PDCA cycle combined with WeChat platform in health education of patients with PICC. J Nurs Train. 2016;31(14):1287-90.

20. Wu KK, Chan SK, Ma TM. Posttraumatic stress, anxiety, and depression in survivors of severe acute respiratory syndrome (SARS). J Trauma Stress. 2005;18(1):39-42. doi:10.1002/jts.20004.

21. Leung GM, Lam TH, Ho LM, Ho SY, Chan BH, Wong IO, Hedley AJ. The impact of community psychological responses on outbreak control for severe acute respiratory syndrome in Hong Kong. J Epidemiol Community Health. 2003;57(11):857-63. Erratum in: J Epidemiol Community Health. 2003;57(12):995. doi:10.1136/jech.57.11.857.

22. Hawryluck L, Gold WL, Robinson S, Pogorski S, Galea S, Styra R. SARS control and psychological effects of quarantine, Toronto, Canada. Emerg Infect Dis. 2004;10(7):1206-12. doi:10.3201/eid1007.030703.

23. Shultz JM, Cooper JL, Baingana F, Oquendo MA, Espinel Z, Althouse BM, et al. The role of fear-related behaviors in the 2013-2016 West Africa ebola virus disease outbreak. Curr Psychiatry Rep. 2016;18(11):104. doi:10.1007/s11920-016-0741-y.

24. Nguyen HC, Nguyen MH, Do BN, Tran CQ, Nguyen TTP, Pham KM, et al. People with suspected COVID-19 symptoms were more likely depressed and had lower health-related quality of life: the potential benefit of health literacy. J Clin Med. 2020;9(4):965. doi:10.3390/jcm9040965. 